\title{
Autophagy: machinery and regulation
}

\author{
Zhangyuan Yin, Clarence Pascual and Daniel J. Klionsky* \\ Life Sciences Institute, and Department of Molecular, Cellular and Developmental Biology; University of Michigan, Ann Arbor, MI, \\ USA 48109. \\ * Corresponding Author: \\ Daniel J. Klionsky, Tel: +1 734615 6556; E-mail: klionsky@umich.edu
}

\begin{abstract}
Macroautophagy/autophagy is an evolutionarily conserved cellular degradation process that targets cytoplasmic materials including cytosol, macromolecules and unwanted organelles. The discovery and analysis of autophagy-related (Atg) proteins have unveiled much of the machinery of autophagosome formation. Although initially autophagy was regarded as a survival response to stress, recent studies have revealed its significance in cellular and organismal homeostasis, development and immunity. Autophagic dysfunction and dysregulation are implicated in various diseases. In this review, we briefly summarize the physiological roles, molecular mechanism, regulatory network, and pathophysiological roles of autophagy.
\end{abstract}

doi: 10.15698/mic2016.12.546

Received originally: 07.09.2016;

in revised form: 07.10.2016,

Accepted 07.10.2016,

Published 01.12.2016.

Keywords: autophagy, autophagosome formation, physiological roles, cellular homeostasis, pathogenesis, physiological roles, regulation.

\author{
Abbreviations: \\ AMPK - AMP-activated protein kinase, \\ Atg - autophagy related, \\ PAS - phagophore assembly site, \\ PKA - protein kinase $A$, \\ PtdIns3K - class III phosphatidylinositol 3- \\ kinase, \\ Ptdlns3P - phosphatidylinositol 3- \\ phosphate, \\ TORC1 - target of rapamycin complex 1 , \\ Ubl-ubiquitin-like.
}

\section{INTRODUCTION}

Cellular homeostasis requires a proper balance between synthesis and degradation. The two major degradative pathways for cellular components in eukaryotic organisms are autophagy and the proteasome. Autophagy ("selfeating") is the bulk degradation of long-lived cytosolic proteins and organelles; whereas the ubiquitin-proteasome degradative system is primarily responsible for the turnover of short-lived proteins. There are distinct types of autophagy, which vary from each other based on the inducing signals and temporal aspects of induction, type of cargo and mechanism of sequestration. One of the fundamental differences between different types of autophagy is that they can be selective or nonselective. Macroautophagy (hereafter autophagy) is the best characterized pathway and involves the formation of a transient doublemembrane structure, the phagophore, that is the active sequestering compartment. Following expansion and closure this structure becomes an autophagosome that subsequently fuses with the lysosome/vacuole leading to the degradation of the cargo $[1,2]$.

In this review, we discuss the physiological roles of autophagy including its function in protein and organelle quality control, development, cell death and immunity. We also describe the morphology and machinery underlying the formation of the autophagosome, which can be summarized in five distinct events: induction, nucleation, expansion, fusion and cargo degradation/recycling. Next, we distinguish among the different mechanisms used to regulate autophagy, and conclude with a brief discussion concerning pathological connections with this process.

\section{PHYSIOLOGICAL ROLES OF AUTOPHAGY}

As a highly conserved survival mechanism of all eukaryotic cells, autophagy primarily acts as an adaptive response to environmental adversity, especially starvation, one of the most common threats to many organisms. When there is no food available, or when resources become limited, cells will start to degrade and recycle macromolecules including proteins, lipids and carbohydrates for the synthesis of essential components and as an energy supply. One of the main mechanisms available for this purpose is autophagy. With the discovery of the autophagy-related (ATG) genes in yeast and subsequent in-depth studies in various animal and cellular models, many additional physiological processes have been linked to autophagy including intracellu- 
lar quality control, maintenance of cellular and tissue homeostasis, anti-aging, cell differentiation and development, and innate and adaptive immunity.

\section{Protein/organelle quality control and cellular homeosta-} sis

Autophagy induced by nutrient deprivation or metabolic perturbations is relatively nonselective, and essentially any part of the cytoplasm can be recycled via this bulk degradative pathway. Conversely, autophagy can be highly selective to facilitate disposal of damaged or surplus structures before they become toxic to the cells [3]. This latter type of autophagy is characterized by the presence of degradation cues, typically including a ligand on the target, and the involvement of selective autophagy receptors along with at least one scaffold protein $[4,5]$. Selective autophagy targets cargoes including protein aggregates, mitochondria, peroxisomes, endoplasmic reticulum, bacterial pathogens and signaling complexes when they are no longer needed [6]. One of the best-characterized selective autophagy-like mechanisms is the yeast cytoplasm-to-vacuole targeting (Cvt) pathway, which utilizes the core machinery of autophagy to deliver the precursor form of the hydrolase aminopeptidase I, along with other degradative enzymes, to the vacuole [7].

Although much attention has been paid to induced autophagy that occurs under different stress conditions, constitutive turnover of cytoplasmic contents by basal autophagy, even during favorable growth conditions, is also crucial for proper cell physiology. This low level of autophagy works in part as a quality-control mechanism, and is especially vital for homeostasis of post-mitotic cells such as hepatocytes and neurons. Genetic studies have revealed that failures in basal autophagy are associated with neurodegenerative disease, cancer and inflammation. For example, mice deficient for ATG7 in pancreatic epithelial cells develop severe pancreatic inflammation and extensive fibrosis [8], neural cell-specific Atg5 knockout mice show accumulation of abnormal proteins in neurons and exhibit deficits in motor function [9], and the decreased expression of mitophagy genes leads to unwarranted inflammation [5]. Therefore, autophagy works as a cellular housekeeper in normal physiological conditions. Accumulation of misfolded and oxidatively damaged proteins, as well as dysfunctional organelles such as mitochondria, is not only a sign of but also one of the causes of aging [10]. Accordingly, the clearance of protein aggregates and improperly functioning organelles helps improve cellular function, extend lifespan and avoid cell death. Indeed, autophagy is suggested to confer anti-aging effects. On the one hand, defective autophagy is associated with degeneration and premature aging. On the other hand, increased autophagy at the whole-body level contributes to longevity in different model organisms [11]. Although the specific mechanism through which autophagy might contribute to antiaging remains unknown, the modulation of this pathway is still considered to be a promising target for improving healthy aging.

\section{Development}

The capability of autophagy to respond to external cues rapidly, and to modify intracellular architecture enables it to be the crucial mechanism for cellular remodeling during animal development [12]. The generation of autophagydefective mutants in various model organisms has shown the important roles autophagy plays during development. For example, autophagy participates in sporulation in yeast, it is necessary for dauer formation and the degradation of $\mathrm{P}$ granules in C. elegans somatic cells, and in Drosophila certain autophagy mutants show larval lethality or failure in metamorphosis [13]. In mammals, autophagy is vital for pre-implantation embryo development, the survival of neonates and organogenesis. After fertilization, autophagy along with the ubiquitin-proteasome system disposes of sperm mitochondria, and thus contributes to heteroplasmy [14]. After the late two-cell stage, autophagy is highly activated, targeting maternal mRNA and proteins, which might be necessary for zygotic genome activation [15]. Atg5 knockout mice with the elimination of maternally derived ATG5 produce embryos that never go beyond the eight-cell stage.

Another example of the role of autophagy at an organismal level can be seen in newborn mice. Following birth, the placental nutrient supply from the mother suddenly terminates, which challenges the neonates with severe starvation; autophagy appears to play a critical role during the transition to breast feeding by supplying nutrients, although it is also possible that neurological defects associated with the absence of autophagy result in an inability to breast feed [16]. During late stages of embryonic and postnatal development, autophagy also plays an important role in cardiogenesis, osteogenesis, central nervous system development and cell differentiation [13]. A representative example of the role of autophagy is seen with erythropoiesis. Mature erythrocytes are generated from erythroblasts and are devoid of most cellular organelles. The elimination of mitochondria, ribosomes and other organelles that are no longer necessary for cellular function is partly dependent on autophagy regulated by multiple modulators [17].

\section{Crosstalk with cell death}

During the development of Drosophila, autophagy is found to facilitate cell death while removing obsolete tissues, suggesting a dual role for this primarily cytoprotective process in physiological conditions. Accordingly, there is extensive crosstalk between autophagy and apoptosis with regard to cell fate determination. Both processes are downstream of common signals, such as those involving TP53/p53 and BH3-only proteins, and they share common regulatory components including $B C L 2$, as well as mutually regulating each other [18]. Autophagy reduces the possibility of apoptosis by carrying out mitophagy, and through the specific targeting and degradation of pro-apoptotic proteins [19]. In turn, the activation of apoptosis inhibits autophagy, as caspases can cleave and inactivate essential autophagy proteins. In some cases, the cleavage of autophagic proteins even converts them into pro-apoptotic proteins [19]. In a simple model, autophagy precedes 
apoptosis as a first response to cellular damage; if unsuccessful in eliminating the damage, autophagy is blocked and apoptosis is induced.

The relationship between these two processes can be highly context dependent. For example, during Drosophila larval metamorphosis, the removal of the salivary gland is dependent on both autophagy-mediated cell death and apoptosis, whereas degradation of the mid-gut only relies on autophagy [20]. During ovary development, caspases are needed for autophagy induction under stress conditions, whereas nurse cell apoptosis requires autophagic clearance of its inhibitor, Bruce [21].

It is not difficult to imagine that autophagy can participate in cell death, especially when the process is purposely dysregulated for therapeutic purposes. However, it is important to note that in most organisms autophagy is primarily a protective response. To demonstrate that autophagy is causative of cell death (and in particular has not been induced during stress to prevent cell death), autophagic cell death, formerly termed type II cell death, it is imperative to show that the cell death was caused by and was dependent on autophagy, meaning that it will be suppressed by the chemical or genetic inhibition of autophagy activity [22]. Examples of autophagic cell death include autosis, which is regulated by the $\mathrm{Na}^{+}, \mathrm{K}^{+}$-ATPase pump and can be induced by starvation and autophagy-inducing peptides [23], and ferroptosis, in which autophagy degrades the cellular iron storage protein ferritin and leads to the accumulation of reactive oxygen species inside the cell and consequently cell death [24].

\section{Immunity}

Considering the function of autophagy in organelle elimination, it is not surprising it has evolved as a primary form of innate immunity against microbial invasion. This cellautonomous defense mechanism, termed xenophagy, is able to selectively recognize intracellular microbes including bacteria, viruses, and protozoa, and target them to the autophagic machinery for degradation [25]. In fact, autophagy plays various roles in immunity including control of the pro-inflammatory response, assisting adaptive immunity through the regulation of immune system development and homeostasis, and in antigen presentation [26]. The involvement of autophagy in multiple diseases will be further discussed in this review.

\section{MORPHOLOGY AND MACHINERY}

One of the most exciting topics in the field of autophagy today is understanding the molecular details of autophagosome biogenesis. In many ways, the sequestration step is the most complex part of autophagy; cytoplasm must be segregated, often in a directed or specific manner, and moved from the intracellular space into the vacuole/lysosome lumen, which corresponds to the extracellular space. Thus, sequestration involves an essentially double-membrane intermediate, the phagophore (Figure 1);

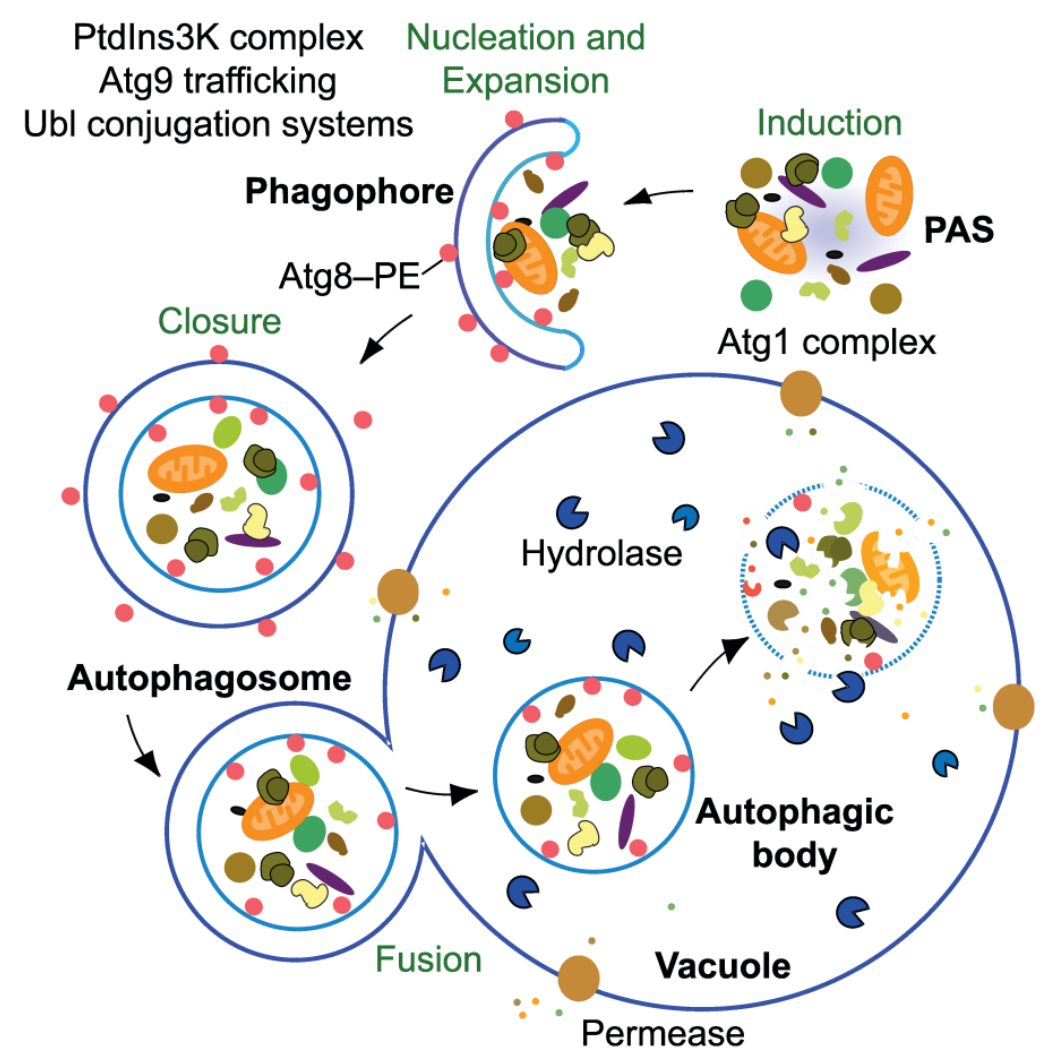

FIGURE 1: The mechanistic features of yeast autophagy. Initiation of autophagy requires the formation of the Atg1 kinase complex at the PAS to allow the recruitment and activation of other Atg proteins. In yeast there is typically one PAS per cell, but the precise nature of this site is not well defined; the PAS may literally become a phagophore, making it a dynamic structure that continuously reforms. Next, the Ptdlns3K complex translocates to the PAS to begin the nucleation process that will catalyze further movement of additional Atg proteins to this site. Ubl proteins such as Atg8-PE participate in cargo recognition during selective types of autophagy, and also play a role in determining the size of the autophagosome. Membrane delivery to the phagophore allows expansion and maturation into an autophagosome. This process also employs the transmembrane protein Atg9; however, the mechanism for phagophore expansion is poorly understood. Upon autophagosome completion, Atg4 deconjugates Atg8-PE on the surface of the autophagosome and the resulting vesicle fuses with the vacuole. In yeast, the inner vesicle is released into the lumen as an autophagic body. Within the vacuole, the contents of the autophagic body are released following lysis by the putative lipase Atg15. Finally, the cargo is degraded by resident hydrolases, and the resulting macromolecules are then released back into the cytosol via permeases. 
the use of a double-membrane structure in effect, changes the topology of the cargo because subsequent fusion releases it into the lumen of the degradative compartment. The formation of the phagophore and its subsequent maturation to become the autophagosome is a transient event, but extremely dynamic, involving multiple protein complexes, that participate in the different stages of autophagy, and the mobilization of substantial membrane reserves. The stages of autophagosome biogenesis can be summarized into five events: induction, nucleation, expansion, fusion and cargo degradation/recycling. In this section of the review, we provide the details for each of these steps and briefly discuss the machinery involved.

\section{Induction and nucleation}

\section{Atg1 kinase complex and induction}

Autophagy may be induced as a response to a change in the extracellular environment of a cell, and the target of rapamycin complex 1 (TORC1) is one of the signaling pathways that plays a primary role in sensing the shift in nutrient availability. Nutrient starvation, in particular nitrogen and/or amino acid limitation, initiates an intracellular signaling cascade by discontinuing TORC1 stimulation, resulting in the activation of the Atg1 kinase complex. The Atg1 kinase complex works directly downstream of the TORC1 pathway and it consists of Atg1, the regulatory protein Atg13, and a scaffold subcomplex that includes Atg17Atg31-Atg29 (Figure 1) [27]. Assembly of this complex is crucial for autophagy because it plays a role in recruiting other Atg proteins to the phagophore assembly site (PAS) and activating downstream targets through phosphorylation $[28,29]$. Protein kinase A (PKA) is another negative regulator of the Atg1 kinase complex, in this case primarily in response to carbon source, whereas the energy sensor Snf1/AMP-activated protein kinase (AMPK) acts as a positive regulator.

\section{Class III phosphotidylinositol 3-kinase (PtdIns3K) complex and nucleation}

In autophagy, nucleation refers to the process of mobilizing a small group of molecules to the PAS; the phagophore is the active sequestering compartment of autophagy. In part, the nucleation process may be viewed as an amplification event that results in the further recruitment of proteins that are needed for phagophore expansion. The class III Ptdlns3K complex I, which is employed specifically for autophagy, is one of the key complexes that are recruited to the PAS upon induction of autophagy. This complex is comprised of five distinct proteins: the lipid kinase Vps34, the regulatory kinase Vps15, Vps30/Atg6, Atg14 and Atg38, which are all necessary for autophagy (Figure 1) [30, 31]. In brief, the class III PtdIns3K is responsible for the production of phosphatidylinositol-3-phosphate (Ptdlns3P) directly from phosphatidylinositol [32]. This Ptdlns3P is important for the correct localization of some of the Atg proteins including Atg18 and Atg2, which enables the recruitment of Atg8, Atg9 and Atg12 to the PAS [33].

\section{Phagophore expansion}

Ubiquitin-like (Ubl) conjugation systems and expansion

A characteristic feature of autophagy is the formation of double-membraned vesicles known as autophagosomes, which correspond to the mature form of the phagophore (Figure 1) [34]. It is important to note that phagophores are relatively transient, even relative to autophagosomes. As a result, much attention has focused on the latter, even though the autophagosome is essentially a terminal compartment that does little more than fuse with the vacuole; in other words, formation of, and sequestration by, the phagophore are the truly dynamic steps of autophagy.

There are two essential ubiquitin-like (Ubl) conjugation systems that are necessary for phagophore expansion and these involve the Ubl proteins Atg12 and Atg8 [35]; these two proteins have structural similarity to ubiquitin, but are not actual homologs. Atg12 is conjugated to Atg5 via the action of the E1 and E2 enzymes Atg7 and Atg10, and this conjugate binds Atg16 to form the dimeric Atg12-Atg5Atg16 complex; there is no known E3 enzyme required for Atg12 conjugation to Atg5. Atg8 undergoes a different type of conjugation, being covalently attached to the lipid phosphatidylethanolamine. The generation of Atg8-PE involves the protease Atg4, Atg7 as an E1 enzyme and Atg3 as an E2 enzyme, with the Atg12-Atg5-Atg16 complex participating as an E3 enzyme [35], although the latter is not absolutely required for conjugation to occur [36]. A detailed mechanism in which these conjugation systems operate along with other complexes to enlarge the phagophore is currently an on-going research topic. Studies have linked Atg2 and Atg18 to the proper recruitment of both Ubl proteins to the PAS, but whether Atg2 and/or Atg18 directly recruit Atg8 and Atg12 is not yet known [37].

\section{Atg9 trafficking}

Unlike other vesicles in the cells, the autophagosome is often referred to as being made de novo. This term is used to distinguish autophagosome formation from vesicle budding, which occurs throughout the secretory pathway and during endocytosis. One useful way to think about the distinction between autophagy and other vesicle-mediated processes is that vesicles used in the secretory pathway are generally of a uniform size and bud off from a pre-existing organelle already containing their cargo. The phagophore may be generated by vesicular addition (although this is a controversial topic), but it may be variable in size, in part depending on the cargo. A common view in the autophagy field is that Atg9 functions in some manner as the membrane transporter for the growing phagophore, but direct evidence or a mechanistic explanation are not available. Nonetheless, Atg9 is a good candidate for this role for multiple reasons. First, Atg9 is the only transmembrane protein that is essential for phagophore expansion [38]. Second, Atg9 is found to be highly mobile in the cytosol upon rapamycin treatment [39]. Third, this protein is capable of binding with itself and appears to transit to the PAS as part of a complex [40]. While none of these studies directly proves the role of Atg9 in membrane shuttling, researchers have begun identifying the machinery that is involved in 
Atg9 trafficking. These components include Atg11, Atg23 and Atg27, which transit along with Atg9 from putative membrane donor sites to the PAS.

\section{Autophagosome targeting, docking and fusion}

Upon completion of the autophagosome, it targets to, tethers/docks and then fuses with the vacuole. This fusion allows the release of the inner autophagosome vesicle into the vacuole lumen where it is now termed an autophagic body. Note that mammalian cell lysosomes are generally smaller than autophagosomes so autophagic bodies are not a general feature of autophagy in most of the more complex eukaryotes. The mechanism that controls the timing of fusion is not known at present; however, there are regulatory mechanisms in place to prevent premature autophagosome fusion with the vacuole, which would prevent delivery of the cargo into the vacuole lumen. For example, Atg8-PE undergoes a secondary cleavage event by Atg4, a cysteine protease that is also required for the early stages of Atg8 conjugation [41]. This cleavage event (termed deconjugation) is necessary for the autophagosome to initiate its fusion with the vacuole [42]. One suggestion is that deconjugation is the trigger for disassembly of the Atg proteins from the completed autophagosome, a step that presumably precedes fusion. Other cellular processes that also deliver their cargo to the vacuole employ similar components that facilitate fusion including SNARE proteins and those involved in the homotypic fusion and vacuole protein sorting (HOPS) pathway [43].

\section{Cargo degradation and recycling}

After the cargo is delivered inside the vacuole, the autophagic body membrane is degraded by a putative lipase, Atg15 (Figure 1) [44, 45], followed by cargo degradation by resident hydrolases. Once degraded, the resulting macromolecules are released back into the cytosol through various permeases including Atg22 (Figure 1) [46].

\section{REGULATION OF AUTOPHAGY}

Given the important roles autophagy plays in the maintenance of cellular homeostasis and survival under stress conditions and its involvement in various aspects of animal development and pathophysiology, it is not surprising that autophagy needs to be finely regulated to avoid either excessive or insufficient activity. Numerous studies have been focusing on how autophagy is kept at basal levels in normal conditions and how it is quickly switched on upon certain types of stimulation. Less well understood are the mechanisms that downregulate and prevent excessive autophagy when cells are maintained under stress conditions. Similar to the mechanism of autophagy itself, the regulatory network shows a lot in common across a broad spectrum of organisms from yeast to mammals, although the inducing signals can be more complicated in more complex eukaryotes. Nonetheless, studies in yeast have pioneered our understanding of this regulatory network.

\section{Nitrogen-dependent regulation}

It was known that glucose or amino acid deprivation will induce autophagy long before the identification of the ATG genes $[47,48]$. The primary sensor of amino acid and nitrogen change is TOR or the mammalian homolog MTOR (mechanistic target of rapamycin [serine/threonine kinase]), which is the main negative regulator of autophagy. TOR/MTOR is a conserved serine/threonine kinase that senses and integrates multiple environmental signals to inhibit catabolism and coordinate cell growth. MTORC1 can be activated by cues including energy status, nutrient levels, growth factors and amino acids [49]. For yeast in nutrient-rich conditions, TORC1 directly phosphorylates Atg13, Atg1 and Atg14, which prevents the formation and/or activation of the Atg1-Atg13-Atg17-Atg31-Atg29 complex and suppresses the autophagy-specific Ptdlns3K, thus inhibiting autophagy induction [50,51]. In mammalian cells, amino acids are sensed by the vacuolar-type $\mathrm{H}^{+}$-translocating ATPase, which is present in the lysosome membrane, in conjunction with RRAG proteins and the Ragulator complex [52], which can coordinately direct MTORC1 to the lysosome membrane where it becomes activated by the GTPase RHEB [53]. Under conditions of amino acid withdrawal, or treatment with the inhibitors rapamycin or Torin1, MTORC1 is suppressed and autophagy becomes derepressed. In addition to MTORC1 inactivation, nitrogen starvation results in the dephosphorylation of ULK1 (a homolog of yeast Atg1) through protein phosphatase $2 \mathrm{~A}$ [54].

In addition to these types of post-translational regulation, transcriptional regulators also function in response to nitrogen and amino acid depletion. For example, yeast Gcn2 is a kinase that is able to sense the level of amino acids; once activated, Gcn2 induces the transcription of specific ATG genes including ATG1 through the activation of the transcription factor Gcn4 [55]. In mammals, inactivation of MTORC1 allows the unphosphorylated form of TFEB (transcription factor EB) to translocate to the nucleus, which similarly results in the transcription of various genes involved in autophagic degradation [56].

\section{Energy/glucose-dependent regulation}

Regulation of autophagy by glucose metabolism and energy level is also vital for cellular homeostasis. In the presence of glucose, PKA is activated by binding with CAMP. PKA then phosphorylates Atg1 and Atg13, which prevents the localization of Atg13 to the PAS [57]. In addition, PKA can inhibit autophagy by direct phosphorylation of TORC1 or in mammalian cells by indirect activation of MTORC1 through inhibition of AMPK. AMPK is the major energy sensor in the cell and it is activated by an increased AMP:ATP ratio, which is one outcome of glucose depletion or other types of stress such as mitochondrial dysfunction [58]. When AMPK senses low energy, it promotes autophagy by inhibiting MTOR activity through direct negative phosphorylation of RPTOR/raptor, a subunit of the MTORC1 complex, or phosphorylation and activation of the TSC1/2 complex, a negative regulator of MTORC1 [59]. Moreover, AMPK is able to activate ULK1 by direct phos- 
phorylation [60]. Intriguingly, this activation can be suppressed by MTORC1-dependent phosphorylation of ULK1 and a negative feedback phosphorylation of AMPK by ULK1. Similar to nitrogen deprivation, glucose starvation can activate the transcription of certain ATG genes by deacetylation of transcription factors including FOXO1 and $\mathrm{FOXO} 3$ [61].

\section{Other types of regulation}

Some types of lipid metabolism are closely related to autophagy. Free fatty acids stimulate autophagy through EIF2AK2/PKR-dependent activation of EIF2S1/elF2 $\alpha$, MAPK8 (mitogen-activated protein kinase 8 ) or inhibition of MTORC1. Dietary lipid existing in the form of intracellular droplets will induce autophagy and be captured and delivered to lysosomes for degradation. The breakdown product, in particular fatty acids, will then mediate autophagy to avoid potential lipotoxicity [62].

Additional stimuli that are capable of inducing autophagy include hypoxia, ER stress, the amino acid metabolite ammonium, depletion of iron and the absence of growth factors. These environmental cues and relevant pathways can be more interconnected and complex at the tissue or body level. To further explore the downstream targets of the master regulators such as MTORC1 and how each ATG protein is specifically regulated, researchers have started to screen and analyze the transcription factors involved in autophagy regulation. Several transcription factors have been identified and characterized. For example, in yeast Ume6 negatively regulates ATG8 and the inhibitory phosphorylation of Ume6 is partly responsible for the dramatic increase of ATG8 transcripts upon starvation, which in turn controls the size of autophagosomes [63]. Pho23 controls ATG9 transcript levels, thereby regulating the number of autophagosomes [64].

\section{PATHOLOGY}

As discussed above, autophagy plays critical roles in maintaining normal cellular physiology. Accordingly, it is not surprising that defects in autophagy have been linked to a wide range of diseases $[65,66]$. In fact, autophagy plays such a fundamental role in cellular health that defects in this process have been linked a vast array of pathophysiologies, which are beyond the scope of this review. Here, we briefly highlight one example of the connection between autophagy and neurodegeneration, which illustrates the complexities of manipulating this process for disease treatment.

Autophagic dysfunction has been associated with a large number of neurodegenerative diseases. One of the hallmarks of many neurodegenerative diseases is the accumulation of aggregated proteins that escape the degradative process resulting in neuronal cell death [67, 68]. One of the basic concepts is that fully differentiated nondividing cells rely extensively on autophagy to remove waste products that accumulate over time. Thus, neurons are particularly dependent on autophagic degradation. Most neurodegenerative diseases are age related, making it dif- ficult to demonstrate a direct correlation between defective autophagy and the disease phenotype; however, genetically impaired autophagic flux in the central nervous system of otherwise healthy mice results in symptoms of neurodegeneration, suggesting a direct link between the two $[9,69]$.

Alzheimer disease is one example of neurodegeneration associated with autophagy. Amyloid plaques composed of amyloid- $\beta$ peptides and hyperphosphorylated MAPT/tau proteins are the most commonly known marker of the disease [70]; although, as with most neurodegenerative diseases, the actual cause of toxicity is not known. Nonetheless, a general model is that defects in autophagy, which generally declines with age, may lead to an increase in these neuropeptides, ultimately resulting in neuronal cell death. Along these lines, autophagosomes have been seen to accumulate in samples from patients with Alzheimer disease, which suggests a block in the degradative system [71]. Conversely, it has been proposed that in diseased cells the mammalian autophagosome may actually contribute to the generation of disease-causing peptides [72], implicating autophagy as playing a more active role in disease onset. Thus, this observation provides one example of the basic conundrum of attempting to manipulate autophagy. That is, autophagy is essential, yet too much autophagy can be lethal. Similarly, the induction of autophagy in aged organisms may have beneficial consequences, but there is also the possibility that it can promote certain disease conditions. Thus, until we know much more about this process, we must be extremely cautious in the use of autophagy-modulating drugs in disease treatment.

\section{CONCLUDING REMARKS}

Autophagy, a tightly regulated intracellular degradation process, is crucial for cellular homeostasis, survival and organismal development, and autophagic dysfunction is linked to numerous diseases. Despite the tremendous progress made in the last two decades, our understanding of autophagy is still relatively primitive and there are many questions remaining to be answered.

During autophagy, various autophagy-related proteins undergo post-translational modification. However, how this regulation affects the overall activity of selective autophagy remains unclear. In recent years, researchers have discovered several forms of noncanonical autophagy, which only needs a subset of ATG proteins and bypasses some of the otherwise "essential" complexes. In this regard, it will be intriguing to know what the relevance is among these different mechanisms and, along a related line, how the ATG proteins gained pleiotropic functions. Some detailed aspects of autophagy also need further investigation. For example, what are the sources of the phagophore membrane? How is membrane incorporated into the expanding phagophore? Is there any type of coordinate regulation among donor membrane sources? Additional studies on the structure and interactions of the autophagy-related proteins might shed light on the above questions. 
Another key question is how autophagy machinery exhibits cell- or tissue-type specificity. Each cell type has its own structural and functional characteristics and they also may require autophagy in different developmental contexts. For example, red blood cells and neurons both rely on autophagy, but for different purposes and at different times of development. How is autophagy differentially regulated in different cell types to allow proper cell differentiation and to maintain homeostasis of cells experiencing variable environments? How is the crosstalk among different regulatory pathways initiated by distinct stimuli and how is regulation coordinated? In addition, when studying the regulatory network in transformed cell lines, there is a concern that the artificial stress conditions might fail to adequately mimic the true physiological conditions. Therefore, establishing a method to monitor autophagy activity in vivo in a real-time manner and generating specific chemicals to inhibit or induce autophagy without apparent deleterious side effects will be particularly significant. Undoubtedly, an improved understanding of autophagy will

\section{REFERENCES}

1. Klionsky DJ, Emr SD (2000). Autophagy as a regulated pathway of cellular degradation. Science 290(5497): 1717-1721.

2. Parzych KR, Klionsky DJ (2014). An overview of autophagy: morphology, mechanism, and regulation. Antioxid Redox Signal 20(3): 460-473.

3. Johansen T, Lamark T (2011). Selective autophagy mediated by autophagic adapter proteins. Autophagy 7(3): 279-296.

4. Mijaljica D, Nazarko TY, Brumell JH, Huang W-P, Komatsu M, Prescott M, Simonsen A, Yamamoto A, Zhang H, Klionsky DJ, Devenish RJ (2012). Receptor protein complexes are in control of autophagy. Autophagy 8(11): 1701-1705.

5. Okamoto $\mathrm{K}$ (2014). Organellophagy: eliminating cellular building blocks via selective autophagy. J Cell Biol 205(4): 435-445.

6. Khaminets A, Behl C, Dikic I (2016). Ubiquitin-Dependent And Independent Signals In Selective Autophagy. Trends Cell Biol 26(1): 616.

7. Lynch-Day MA, Klionsky DJ (2010). The Cvt pathway as a model for selective autophagy. FEBS Lett 584(7): 1359-1366.

8. Antonucci L, Fagman JB, Kim JY, Todoric J, Gukovsky I, Mackey M, Ellisman $\mathrm{MH}$, Karin $\mathrm{M}$ (2015). Basal autophagy maintains pancreatic acinar cell homeostasis and protein synthesis and prevents ER stress. Proc Natl Acad Sci U S A 112(45): E6166-6174.

9. Hara T, Nakamura K, Matsui M, Yamamoto A, Nakahara Y, SuzukiMigishima R, Yokoyama M, Mishima K, Saito I, Okano H, Mizushima N (2006). Suppression of basal autophagy in neural cells causes neurodegenerative disease in mice. Nature 441(7095): 885-889.

10. Terman A, Gustafsson B, Brunk UT (2007). Autophagy, organelles and ageing. J Pathol 211(2): 134-143.

11. Rubinsztein DC, Marino G, Kroemer G (2011). Autophagy and aging. Cell 146(5): 682-695.

12. Mizushima N, Levine B (2010). Autophagy in mammalian development and differentiation. Nat Cell Biol 12(9): 823-830.

13. Di Bartolomeo S, Nazio F, Cecconi F (2010). The role of autophagy during development in higher eukaryotes. Traffic 11(10): 1280-1289. provide more therapeutic targets for pharmaceutical or genetic strategies against disease.

\section{ACKNOWLEDGMENTS}

This work was supported by NIH grant GM053396 to DJK.

\section{CONFLICT OF INTEREST}

The authors declare no conflict of interest.

\section{COPYRIGHT}

(C) 2016 Yin et al. This is an open-access article released under the terms of the Creative Commons Attribution (CC BY) license, which allows the unrestricted use, distribution, and reproduction in any medium, provided the original author and source are acknowledged.

Please cite this article as: Zhangyuan Yin, Clarence Pascual and Daniel J. Klionsky (2016). Autophagy: machinery and regulation. Microbial Cell 3(12): 588-596. doi: 10.15698/mic2016.12.546

14. Song WH, Yi YJ, Sutovsky M, Meyers S, Sutovsky $P$ (2016). Autophagy and ubiquitin-proteasome system contribute to sperm mitophagy after mammalian fertilization. Proc Natl Acad Sci U S A

15. Stitzel ML, Seydoux G (2007). Regulation of the oocyte-to-zygote transition. Science 316(5823): 407-408.

16. Kuma A, Hatano $M$, Matsui $M$, Yamamoto A, Nakaya $H$, Yoshimori $\mathrm{T}$, Ohsumi $Y$, Tokuhisa T, Mizushima N (2004). The role of autophagy during the early neonatal starvation period. Nature 432(7020): 10321036 .

17. Mortensen M, Ferguson DJ, Edelmann M, Kessler B, Morten KJ, Komatsu M, Simon AK (2010). Loss of autophagy in erythroid cells leads to defective removal of mitochondria and severe anemia in vivo. Proc Natl Acad Sci U S A 107(2): 832-837.

18. Denton D, Xu T, Kumar S (2015). Autophagy as a pro-death pathway. Immunol Cell Biol 93(1): 35-42.

19. Marino G, Niso-Santano M, Baehrecke EH, Kroemer G (2014). Selfconsumption: the interplay of autophagy and apoptosis. Nat Rev Mol Cell Biol 15(2): 81-94.

20. Denton D, Shravage B, Simin R, Mills K, Berry DL, Baehrecke EH, Kumar S (2009). Autophagy, not apoptosis, is essential for midgut cell death in Drosophila. Curr Biol 19(20): 1741-1746.

21. Zhang $H$, Baehrecke EH (2015). Eaten alive: novel insights into autophagy from multicellular model systems. Trends Cell Biol 25(7): 376-387.

22. Galluzzi L, Vitale I, Abrams JM, Alnemri ES, Baehrecke EH, Blagosklonny MV, Dawson TM, Dawson VL, El-Deiry WS, Fulda S, Gottlieb E, Green DR, Hengartner MO, Kepp O, Knight RA, Kumar S, Lipton SA, Lu X, Madeo F, Malorni W, Mehlen P, Nunez G, Peter ME, Piacentini M, Rubinsztein DC, Shi Y, Simon HU, Vandenabeele P, White $E$, Yuan J, et al. (2012). Molecular definitions of cell death subroutines: recommendations of the Nomenclature Committee on Cell Death 2012. Cell Death Differ 19(1): 107-120.

23. Liu Y, Levine B (2015). Autosis and autophagic cell death: the dark side of autophagy. Cell Death Differ 22(3): 367-376. 
24. Gao M, Monian P, Pan Q, Zhang W, Xiang J, Jiang X (2016). Ferroptosis is an autophagic cell death process. Cell Res.

25. Deretic V, Levine B (2009). Autophagy, immunity, and microbial adaptations. Cell Host Microbe 5(6): 527-549.

26. Deretic V, Saitoh T, Akira S (2013). Autophagy in infection, inflammation and immunity. Nat Rev Immunol 13(10): 722-737.

27. Kamada Y, Funakoshi T, Shintani T, Nagano K, Ohsumi M, Ohsumi Y (2000). Tor-mediated induction of autophagy via an Apg1 protein kinase complex. J Cell Biol 150(6): 1507-1513.

28. Suzuki K, Kubota Y, Sekito T, Ohsumi Y (2007). Hierarchy of Atg proteins in pre-autophagosomal structure organization. Genes Cells 12(2): 209-218.

29. Papinski D, Schuschnig $M$, Reiter W, Wilhelm L, Barnes CA, Maiolica A, Hansmann I, Pfaffenwimmer T, Kijanska M, Stoffel I, Lee SS, Brezovich A, Lou JH, Turk BE, Aebersold R, Ammerer G, Peter M, Kraft C (2014). Early steps in autophagy depend on direct phosphorylation of Atg9 by the Atg1 kinase. Mol Cell 53(3): 471-483.

30. Schu PV, Takegawa K, Fry MJ, Stack JH, Waterfield MD, Emr SD (1993). Phosphatidylinositol 3-kinase encoded by yeast VPS34 gene essential for protein sorting. Science 260(5104): 88-91.

31. Kihara A, Noda T, Ishihara N, Ohsumi Y (2001). Two distinct Vps34 phosphatidylinositol 3-kinase complexes function in autophagy and carboxypeptidase $\mathrm{Y}$ sorting in Saccharomyces cerevisiae. J Cell Biol 152(3): 519-530.

32. Burman C, Ktistakis NT (2010). Regulation of autophagy by phosphatidylinositol 3-phosphate. FEBS Lett 584(7): 1302-1312.

33. Obara K, Sekito T, Niimi K, Ohsumi Y (2008). The Atg18-Atg2 complex is recruited to autophagic membranes via phosphatidylinositol 3-phosphate and exerts an essential function. J Biol Chem 283(35): 23972-23980.

34. Baba M, Takeshige K, Baba N, Ohsumi Y (1994). Ultrastructural analysis of the autophagic process in yeast: detection of autophagosomes and their characterization. J Cell Biol 124(6): 903913.

35. Ohsumi Y (2001). Molecular dissection of autophagy: two ubiquitin-like systems. Nat Rev Mol Cell Biol 2(3): 211-216.

36. Cao Y, Cheong H, Song H, Klionsky DJ (2008). In vivo reconstitution of autophagy in Saccharomyces cerevisiae. J Cell Biol 182(4): 703-713.

37. Kobayashi T, Suzuki K, Ohsumi Y (2012). Autophagosome formation can be achieved in the absence of Atg18 by expressing engineered PAS-targeted Atg2. FEBS Lett 586(16): 2473-2478.

38. Noda T, Kim J, Huang W-P, Baba M, Tokunaga C, Ohsumi $Y$, Klionsky DJ (2000). Apg9p/Cvt7p is an integral membrane protein required for transport vesicle formation in the Cvt and autophagy pathways. J Cell Biol 148(3): 465-480.

39. Yamamoto H, Kakuta S, Watanabe TM, Kitamura A, Sekito T, Kondo-Kakuta C, Ichikawa R, Kinjo M, Ohsumi Y (2012). Atg9 vesicles are an important membrane source during early steps of autophagosome formation. J Cell Biol 198(2): 219-233.

40. Reggiori F, Shintani T, Nair U, Klionsky DJ (2005). Atg9 cycles between mitochondria and the pre-autophagosomal structure in yeasts. Autophagy 1(2): 101-109.

41. Kirisako $T$, Ichimura $Y$, Okada $H$, Kabeya $Y$, Mizushima N, Yoshimori T, Ohsumi M, Takao T, Noda T, Ohsumi Y (2000). The reversible modification regulates the membrane-binding state of Apg8/Aut7 essential for autophagy and the cytoplasm to vacuole targeting pathway. J Cell Biol 151(2): 263-276.
42. Nair U, Yen W-L, Mari M, Cao Y, Xie Z, Baba M, Reggiori F, Klionsky DJ (2012). A role for Atg8-PE deconjugation in autophagosome biogenesis. Autophagy 8(5): 780-793.

43. Jiang $P$, Nishimura T, Sakamaki Y, Itakura E, Hatta T, Natsume T, Mizushima N (2014). The HOPS complex mediates autophagosomelysosome fusion through interaction with syntaxin 17. Mol Biol Cell 25(8): 1327-1337.

44. Epple UD, Suriapranata I, Eskelinen E-L, Thumm M (2001). Aut5/Cvt17p, a putative lipase essential for disintegration of autophagic bodies inside the vacuole. J Bacteriol 183(20): 5942-5955.

45. Teter SA, Eggerton KP, Scott SV, Kim J, Fischer AM, Klionsky DJ (2001). Degradation of lipid vesicles in the yeast vacuole requires function of Cvt17, a putative lipase. J Biol Chem 276(3): 2083-2087.

46. Yang Z, Huang J, Geng J, Nair U, Klionsky DJ (2006). Atg22 recycles amino acids to link the degradative and recycling functions of autophagy. Mol Biol Cell 17(12): 5094-5104.

47. Deter RL, de Duve C (1967). Influence of glucagon, an inducer of cellular autophagy, on some physical properties of rat liver lysosomes. J Cell Biol 33(2): 437-449.

48. Mortimore GE, Schworer CM (1977). Induction of autophagy by amino-acid deprivation in perfused rat liver. Nature 270(5633): 174176.

49. Laplante M, Sabatini DM (2012). mTOR signaling in growth control and disease. Cell 149(2): 274-293.

50. Kamada Y, Yoshino K, Kondo C, Kawamata T, Oshiro N, Yonezawa $\mathrm{K}$, Ohsumi $Y$ (2010). Tor directly controls the Atg1 kinase complex to regulate autophagy. Mol Cell Biol 30(4): 1049-1058.

51. Yuan HX, Russell RC, Guan K-L (2013). Regulation of PIK3C3/VPS34 complexes by MTOR in nutrient stress-induced autophagy. Autophagy 9(12): 1983-1995.

52. Sancak Y, Bar-Peled L, Zoncu R, Markhard AL, Nada S, Sabatini DM (2010). Ragulator-Rag complex targets mTORC1 to the lysosomal surface and is necessary for its activation by amino acids. Cell 141(2): 290-303.

53. Bar-Peled L, Schweitzer LD, Zoncu R, Sabatini DM (2012). Ragulator is a GEF for the rag GTPases that signal amino acid levels to mTORC1. Cell 150(6): 1196-1208.

54. Wong PM, Feng $Y$, Wang J, Shi R, Jiang $X$ (2015). Regulation of autophagy by coordinated action of $\mathrm{mTORC} 1$ and protein phosphatase 2A. Nat Commun 6:8048.

55. B'Chir W, Maurin AC, Carraro V, Averous J, Jousse C, Muranishi Y, Parry L, Stepien G, Fafournoux P, Bruhat A (2013). The elF2alpha/ATF4 pathway is essential for stress-induced autophagy gene expression. Nucleic Acids Res 41(16): 7683-7699.

56. Settembre C, Di Malta C, Polito VA, Garcia Arencibia M, Vetrini F, Erdin S, Erdin SU, Huynh T, Medina D, Colella P, Sardiello M, Rubinsztein DC, Ballabio A (2011). TFEB links autophagy to lysosomal biogenesis. Science 332(6036): 1429-1433.

57. Stephan JS, Yeh YY, Ramachandran V, Deminoff SJ, Herman PK (2009). The Tor and PKA signaling pathways independently target the Atg1/Atg13 protein kinase complex to control autophagy. Proc Natl Acad Sci U S A 106(40): 17049-17054.

58. Hardie DG, Ross FA, Hawley SA (2012). AMPK: a nutrient and energy sensor that maintains energy homeostasis. Nat Rev Mol Cell Biol 13(4): 251-262.

59. Russell RC, Yuan HX, Guan KL (2014). Autophagy regulation by nutrient signaling. Cell Res 24(1): 42-57. 
60. Kim J, Kundu M, Viollet B, Guan KL (2011). AMPK and mTOR regulate autophagy through direct phosphorylation of Ulk1. Nat Cell Biol 13(2): 132-141.

61. Feng Y, Yao Z, Klionsky DJ (2015). How to control self-digestion: transcriptional, post-transcriptional, and post-translational regulation of autophagy. Trends Cell Biol 25(6): 354-363.

62. Kim KH, Lee MS (2014). Autophagy--a key player in cellular and body metabolism. Nat Rev Endocrinol 10(6): 322-337.

63. Backues SK, Lynch-Day MA, Klionsky DJ (2012). The Ume6-Sin3Rpd3 complex regulates ATG8 transcription to control autophagosome size. Autophagy 8(12): 1835-1836.

64. Jin M, He D, Backues SK, Freeberg MA, Liu X, Kim JK, Klionsky DJ (2014). Transcriptional regulation by Pho23 modulates the frequency of autophagosome formation. Curr Biol 24(12): 1314-1322.

65. Jiang P, Mizushima N (2014). Autophagy and human diseases. Cell Res 24(1): 69-79.

66. Huang J, Klionsky DJ (2007). Autophagy and human disease. Cell Cycle 6(15): 1837-1849.
67. Nixon RA (2005). Endosome function and dysfunction in Alzheimer's disease and other neurodegenerative diseases. Neurobiol Aging 26(3): 373-382.

68. Shibata M, Lu T, Furuya T, Degterev A, Mizushima N, Yoshimori T, MacDonald M, Yankner B, Yuan J (2006). Regulation of intracellular accumulation of mutant Huntingtin by Beclin 1. J Biol Chem 281(20): 14474-14485.

69. Komatsu M, Kominami E, Tanaka K (2006). Autophagy and neurodegeneration. Autophagy 2(4): 315-317.

70. Holtzman DM, Morris JC, Goate AM (2011). Alzheimer's disease: the challenge of the second century. Sci TransI Med 3(77): 77sr71.

71. Nixon RA, Wegiel J, Kumar A, Yu WH, Peterhoff C, Cataldo A, Cuervo AM (2005). Extensive involvement of autophagy in Alzheimer disease: an immuno-electron microscopy study. J Neuropathol Exp Neurol 64(2): 113-122.

72. Yu WH, Cuervo AM, Kumar A, Peterhoff CM, Schmidt SD, Lee JH, Mohan PS, Mercken M, Farmery MR, Tjernberg LO, Jiang Y, Duff K, Uchiyama Y, Naslund J, Mathews PM, Cataldo AM, Nixon RA (2005). Macroautophagy--a novel Beta-amyloid peptide-generating pathway activated in Alzheimer's disease. J Cell Biol 171(1): 87-98. 